Supporting Information

\title{
Surface-Enhanced Raman Scattering for Direct Protein Function Investigation: Controlled Immobilization and Orientation
}

\author{
Hao $\mathrm{Ma}^{1}$, Xiaofan Tang 2 , Yawen Liu ${ }^{1}$, Xiao Xia Han ${ }^{1 *}$, Chengyan $\mathrm{He}^{3}$, Hui Lu${ }^{2 *}$ and Bing Zhao ${ }^{*}$
}

1. State Key Laboratory of Supramolecular Structure and Materials, Jilin University, Changchun 130012, P. R. China.

2. School of Biological Sciences, Faculty of Biology, Medicine and Health, The University of Manchester, Manchester M13 9PT, UK.

3. China-Japan Union Hospital of Jilin University, Changchun 130033, P. R. China.

*Corresponding authors: hanxiaoxia@jlu.edu.cn (X.X.H.); Hui.Lu@manchester.ac.uk (H.L.); zhaob@jlu.edu.cn (B.Z.)

\section{Table of Contents}

Supplementary experimental details: Purification of Erv1C; Preparation of self-assembled silver chips, Archoring IDA and NTA on the chips surface; Preparation of IDA and NTA with 3-MPA; Bonding with peptides and proteins; Catalysis of Cyt c.

\section{Supplementary Tables}

Table S1. Peptides used in this study.

Table S2. Assignments of peptides with IDA.

Table S3. Raman/SERS band assignments of Erv1C and Cyt c.

\section{Supplementary Figures}

Figure S1. SEM image of the self-assembled silver chip.

S5

Figure S2. Design and assembly of spacer molecules and the corresponding SERS spectra.

S6

Figure S3. XPS spectra of Spacer a-modified Ag with $\mathrm{Ni}^{2+} \& \mathrm{HF}$ : (A) full-range; (B) Ni 2p.

Figure S5. XPS spectra of spacer c-modified Ag with $\mathrm{Ni}^{2+} \& \mathrm{HF}$ : (A) full-range; (B) Ni 2p.

Figure S7. XPS spectra of spacer e-modified Ag with $\mathrm{Ni}^{2+} \& \mathrm{HF}$ : (A) full-range; (B) Ni 2p.

Figure S8 \& S9. ${ }^{1} \mathrm{H}$ NMR of compound 1 and 2 in Figure 1.

Figure S11. Tricine SDS-PAGE of Erv1c affinity purification visualized using Coomassie-blue staining (A) and (B) size exclusion chromatography of Erv1C.

Figure S12. Catalytic properties of the elution of Erv1C from the IDA-modified chips.

Figure S13. Investigation of AFP-ATRA interactions using IDA-SS. SERS spectra of (a) ATRA, (b) AFP and (c) AFP with ATRA on the IDA-modified Ag substrates.

Figure S14. Recycling the IDA-modified Ag substrate for 4 times. SERS spectrum of HF followed by those SERS spectra of 4 His-tagged peptides one after another 


\section{Experimental details}

\section{Materials.}

$\mathrm{N}_{\alpha}, \mathrm{N}_{\alpha}$-bis(carboxymethyl)-L-lysine hydrate, Iminodiacetic acid, $\mathrm{AgNO}_{3}$, sodium citrate, 3-Mercaptopropionic acid and poly(diallyldimethylammonium chloride) ( $\mathrm{Mw}=200,000-350,000$, $20 \mathrm{wt} \%$ aqueous solution) were obtained from Sigma-Aldrich. N-(2-Acetamido) iminodiacetic acid, (2S,3S)-1,4-Bis-sulfanylbutane-2,3-diol (DTT) and $\mathrm{CS}_{2}$ were purchased from Aladdin Co., Ltd. Phosphate buffered saline $(0.1 \mathrm{M}, \mathrm{pH}=8)$ and $\mathrm{PD}-10$ desalting columns from GE Healthcare were obtained from Beijing Dingguo Changsheng Biotechnology Co., Ltd. The phosphate buffered saline (PBS; 0.1 M, pH 7.2) used in this study contained $0.8 \% \mathrm{NaCl}, 0.02 \% \mathrm{KH}_{2} \mathrm{PO}_{4}, 0.02 \% \mathrm{KCl}$, and $0.12 \%$ $\mathrm{Na}_{2} \mathrm{HPO}_{4} \cdot 12 \mathrm{H}_{2} \mathrm{O}$. All chemicals were analytical-grade reagents and used without further purification. Milli-Q water was used in the study. All peptides are purchased from Zhejiang Ontores Biotechnologies Co., Ltd.

\section{Purification of Erv1C}

The pET-24a $(+)$ plasmid containing the C-terminal domain of ERV1 gene (Erv1C aa 84-189) was expressed in and purified from Escherichia coli Rosetta-gamiт 2 cells (Novagen) using the method described in (ref 22). Briefly, expression of the LE(H)6-tagged Erv1C proteins was induced by addition of $0.5 \mathrm{mM}$ IPTG (ForMedium) for $16 \mathrm{~h}$ at $16^{\circ} \mathrm{C}$. Cells were harvested and resuspended in binding buffer (40 mM imidazole, $50 \mathrm{mM}$ Tris/ $\mathrm{HCl}$ and $150 \mathrm{mM} \mathrm{NaCl}, \mathrm{pH} \mathrm{7.4)}$ and complemented again with $10 \mu \mathrm{M}$ FAD. Cells were disrupted by sonication in the presence of three EDTA-free protease inhibitor cocktail tablets (Roche) and the cell lysate was centrifuged at $40 \mathrm{k}$ g for $1 \mathrm{~h}$ at $4^{\circ} \mathrm{C}$. The supernatant was added to a column containing $\mathrm{Ni}-\mathrm{NTA}\left(\mathrm{Ni}^{2+}\right.$-nitrilotriacetate $)$ His-Binding beads (Novagen) equilibrated with binding buffer. After wash, the bound LE(H)6-tagged Erv1C proteins were eluted with elution buffer $(500 \mathrm{mM}$ imidazole, $50 \mathrm{mM}$ Tris/ $\mathrm{HCl}$ and $150 \mathrm{mM} \mathrm{NaCl}$, $\mathrm{pH}$ 7.4) and an extra $100 \mu \mathrm{M}$ of FAD were added, and were divided into om5 $\mathrm{ml}$ aliquots, snap-frozen with liquid nitrogen and kept at $-80^{\circ} \mathrm{C}$ until use. Further purification was done by size-exclusion chromatography (SEC) using buffer AE (BAE: $50 \mathrm{mM}$ Tris/HCl, $150 \mathrm{mM} \mathrm{NaCl}$ and 1 mM EDTA, pH 7.4) on a Superdex 200 100/300 GL column (GE Healthcare).

\section{Instrumental}

SERS spectra were collected by a Jobin Yvon/HORIBA LabRam ARAMIS Raman spectrometer equipped with a HeNe laser $(632.8 \mathrm{~nm})$. The typical exposure time for each measurement applied in this work was $1 \mathrm{~s}$ with one-time accumulation. The ultraviolet-visible (UV-vis) spectra of the samples were obtained with a SHIMADTU ultraviolet spectrophotometer (UV-3600). The NMR spectra were obtained on a Bruker AVANCEIII500 system. The X-ray photoelectron spectra (XPS) were acquired with a Thermo ESCALAB 250 photoelectron spectrometer with Al Ka X-ray radiation. Size exclusion chromatography (SEC) by an AKTA Purifier FPLC system (Amersham Pharmacia) operated by Unicorn software. A Superdex 200 increased 10/30 column (Amersham Pharmacia) was used for size exclusion.

\section{Preparation of self-assembled silver chips}


Ag hydrosol was synthesized by a conventional synthetic route reported by Lee and Meisel method. In brief, $36 \mathrm{mg}$ of $\mathrm{AgNO}_{3}$ was dissolved in $200 \mathrm{~mL}$ of water (1.0mM aqueous), and then the solution was heated to $98{ }^{\circ} \mathrm{C}$ with rapid stirring under reflux. A $4 \mathrm{~mL}$ solution of $1 \%$ sodium citrate was added to the solution, and the solution was boiled for $40 \mathrm{~min}$. The Ag nanoparticles with the diameter of 60-70 $\mathrm{nm}$ were prepared. Subsequently, glass slides were immersed in a boiling solution prepared by mixing $30 \% \mathrm{H}_{2} \mathrm{O}_{2}$ and $98 \% \mathrm{H}_{2} \mathrm{SO}_{4}$ with a volume ratio of $3: 7$. After rinsing, the hydroxylated glass slides were obtained. Then they were soaked in a $0.5 \%$ PDDA solution for 40 min. After repeated rinsing with water and drying with nitrogen gas, the PDDA-coated slides were soaked in silver hydrosol for $4 \mathrm{~h}$. Eventually, a layer of Ag NPs was assembled on the glass surface by electrostatic interaction.

\section{Archoring IDA and NTA on the chips surface (Figure $S 2$ a and b)}

Forming dithiocarbamate-anchored monolayer was following Zhu et al work. ${ }^{[1]}$ Briefly, a 25-mM solution of DTC was prepared using two or three equivalents of amine dissolved in PBS, with one equivalent serving as base. The solution was degassed for several minutes with $\mathrm{N}_{2}$ then treated with one equivalent of $\mathrm{CS}_{2}$, agitated with a vortex mixer for $30 \mathrm{~s}$, and left to sit for $2 \mathrm{~h}$ at room temperature in a capped vial. Then immersing silver chips into vial for $4 \mathrm{~h}$ or discoloration. The chips were then washed with water, incubated for $30 \mathrm{~min}$ in a $5 \mathrm{~g} / \mathrm{L}$ aqueous solution of $\mathrm{NiCl}_{2} \cdot 6 \mathrm{H}_{2} \mathrm{O}$, washed with water, and dried in a flow of $\mathrm{N}_{2}$ before protein immobilization and SERS characterization.

\section{Preparation of IDA and NTA with 3-MPA (Figure S2 $\mathbf{c}$ and d)}

The method was following Wasserberg et al work. ${ }^{[2]}$ Briefly, the chips in step 3 were subsequently immersed in a solution of $2 \mathrm{mM}$ MUA in 1:1 EtOH/water and left at least $16 \mathrm{~h}$ under ambient conditions to react. To remove any excess MUA, the samples were rinsed thoroughly with water three times and dried in a flow of $\mathrm{N}_{2}$. The resulting carboxylic acid-terminated monolayers were activated using a freshly prepared solution of $300 \mathrm{mM}$ NHS and $40 \mathrm{mM} \mathrm{N}$-(dimethyl aminopropyl)-N'-ethylcarbodiimide (EDC) in $0.1 \mathrm{M}$ PBS, $\mathrm{pH} 8.0$, for at least 30 min under ambient conditions. After the reaction, the samples were rinsed with water and dried in a flow of $\mathrm{N}_{2}$, and the activated ester-terminated monolayers were immediately reacted with a $1 \mathrm{mM}$ solution of $\mathrm{N} \alpha, \mathrm{N} \alpha$-bis(carboxymethyl)-L-lysine hydrate in $0.1 \mathrm{M} \mathrm{PBS}, \mathrm{pH} 8.0$, for $2 \mathrm{~h}$ under ambient conditions. Chips were then washed with water, incubated for $30 \mathrm{~min}$ in a $5 \mathrm{~g} / \mathrm{L}$ aqueous solution of $\mathrm{NiCl}_{2} \cdot 6 \mathrm{H}_{2} \mathrm{O}$, washed with water, and dried in a flow of $\mathrm{N}_{2}$ before protein immobilization and SERS characterization.

\section{Bonding with peptides and proteins}

Subsequently, the proteins were immobilized by pipetting $500 \mu \mathrm{L}$ of proteins solution at $1 \mathrm{mg} / \mathrm{mL}$ onto chips. The reaction was allowed to progress overnight at $4{ }^{\circ} \mathrm{C}$ or for $4 \mathrm{~h}$ at $37{ }^{\circ} \mathrm{C}$. The chips were rinsed three times with PBS and stored in the plastic centrifuge tube under a moist atmosphere.

\section{Catalysis of Cyt c}

To confirm the preserved catalysis properties of Erv1C after elution, reduced of Cyt $\mathrm{c}$ was used as an alternative to oxygen to study the activity of Erv1C. ${ }^{[3]}$ In brief, final concentrations after mixing of 3 $\mu \mathrm{M}$ Erv1C, $100 \mu \mathrm{M}$ cyt $\mathrm{c}$ and $1 \mathrm{mM}$ DTT were used. It should be note that the buffer should be 
degassed where the oxygen concentration is kept very low.

\section{Protocols of IMAC}

8-1. Assembling IDA onto the silver chips

Briefly, the chips in step 3 were subsequently immersed in a solution of $2 \mathrm{mM}$ IDA-IDA in water and left at least $16 \mathrm{~h}$ under ambient conditions to react. To remove any excess IDA-IDA, the samples were rinsed thoroughly with water three times and dried in a flow of $\mathrm{N}_{2}$.

8-2. Charge the chips and immobilization of the protein: immersing the chips with following aqueous solution at $4{ }^{\circ} \mathrm{C}$ : Firstly, immerse the chips with $400 \mu \mathrm{L} \mathrm{NiSO}_{4}(100 \mathrm{mM})$ for $1 \mathrm{~h}$, and wash the chips with MQ water three times. Then, immerse the chips into the tube containing POI overnight. After washed with MQ water three times, do the SERS test without any other treatment.

Table S1. 7 Peptides used in this study.

\begin{tabular}{lll}
\hline Core level & Sequences & $\mathrm{Mw}$ \\
\hline H & HHHHHH & 840.86 \\
HW & HHHHHHWWWWWW & 1958.14 \\
HF & HHHHHHFFFFFF & 1723.92 \\
HY & HHHHHHYYYYYY & 1819.91 \\
HN & HHHHHHNNNNNN & 1525.48 \\
HG & HHHHHHGGGGGG & 1183.17 \\
HFYW & HHHHHHFFYYWW & 1833.99 \\
\hline
\end{tabular}

H: Histidine; W: Tryptophan; F: Phenylalanine; Y: Tyrosine; N: Asparagine; G: Glycine

Table S2. Assignments of peptides with IDA.

\begin{tabular}{cc}
\hline Raman shift $\left(\mathrm{cm}^{-1}\right)$ & Band Assignments \\
\hline 221 & Ag-S stretching \\
415 & Coordinate bond of N-Ni2 ${ }^{+}$ \\
635 & S-C stretching of IDA \\
980 & C-C stretching of tyrosine \\
997 & Ring breathing of imidazole \\
1000 & Ring breathing of phenylalanine \\
1009 & indole ring of tryptophan \\
1072 & C-C stretching of IDA \\
$1122 / 1347$ & Imide III \\
1443 & C-N stretching of imidazole \\
1536 & Ring breathing of indole ring \\
1547 & Ring breathing of phenylalanine \\
1560 & Ring breathing of tryptophan \\
1572 & Ring breathing of imidazole \\
1581 & C-H bending of IDA \\
1596 & Ring breathing of phenylalanine \\
1600 & Ring vibration \& N-H in-plane vibration
\end{tabular}


Table S3. Raman/SERS band assignments of Erv1C and Cyt c.

\begin{tabular}{llll}
\hline \multicolumn{2}{c}{ Erv1C } & \multicolumn{2}{c}{ Cyt c } \\
Raman shift $\left(\mathrm{cm}^{-1}\right)$ & Band Assignments & Raman shift $\left(\mathrm{cm}^{-1}\right)$ & Band \\
& & & Assignments \\
\hline 1625 & $v\left(\mathrm{C}_{8} \mathrm{C}_{9}\right), v\left(\mathrm{C}_{9} \mathrm{C}_{9 \mathrm{a}}\right)$ & 1583 & $v\left(\mathrm{C}_{\alpha} \mathrm{C}_{\mathrm{m}}\right)_{\text {asym }}$ \\
1575 (ox); 1582 (red) & $v\left(\mathrm{C}_{10 \mathrm{a}} \mathrm{N}_{1}\right), v\left(\mathrm{~N}_{10} \mathrm{C}_{10 \mathrm{a}}\right)$ & 1314 & $\delta\left(\mathrm{C}_{\mathrm{m}} \mathrm{H}\right)$ \\
1459 & $v\left(\mathrm{C}_{7} \mathrm{C}_{8}\right), v\left(\mathrm{C}_{8} \mathrm{Me}\right)$ & 1130 & $v(\text { pyr half-ring })_{\text {sym }}$ \\
1405 & $v\left(\mathrm{~N}_{3} \mathrm{C}_{2}\right), v\left(\mathrm{~N}_{1} \mathrm{C}_{2}\right)$ & 750 & $v($ pyr breathing $)$ \\
1348 & $v\left(\mathrm{~N}_{10} \mathrm{C}_{10 \mathrm{a}}\right), v\left(\mathrm{C}_{5 \mathrm{a}} \mathrm{C}_{6}\right)$ & & \\
\hline
\end{tabular}

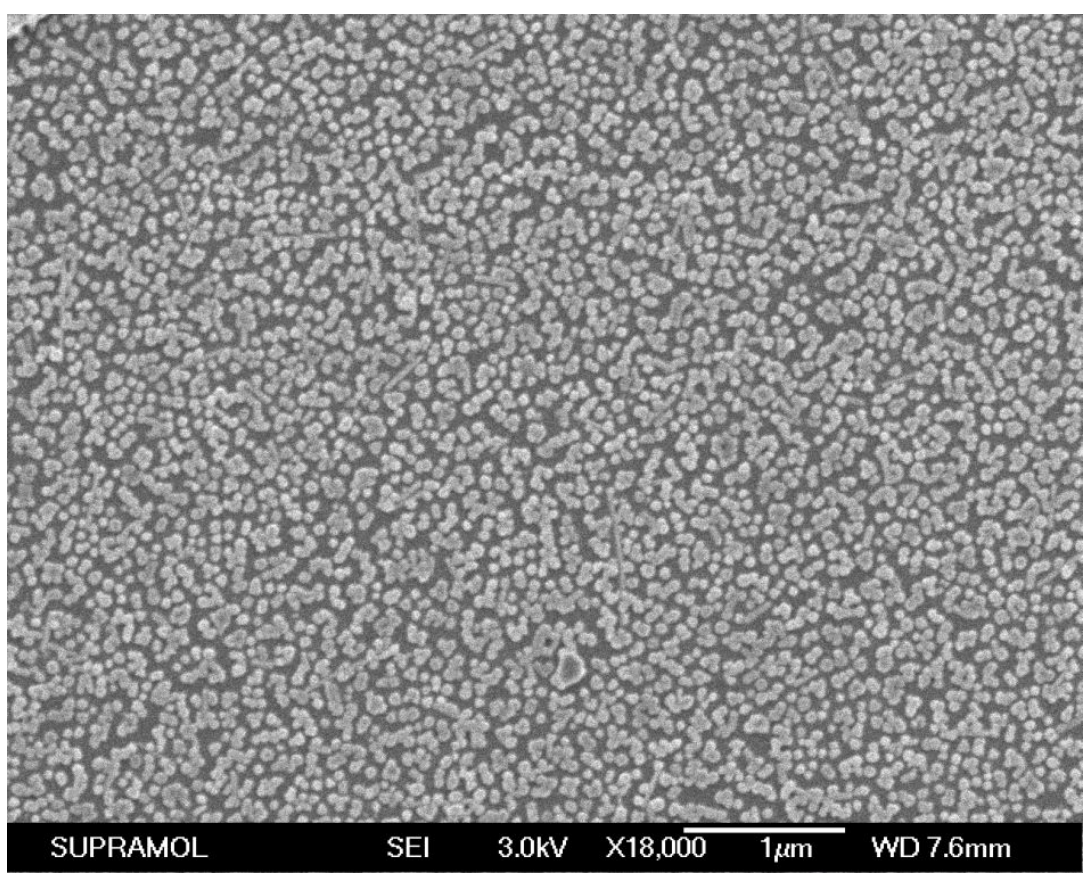

Figure S1. SEM image of the self-assembled silver chip. 

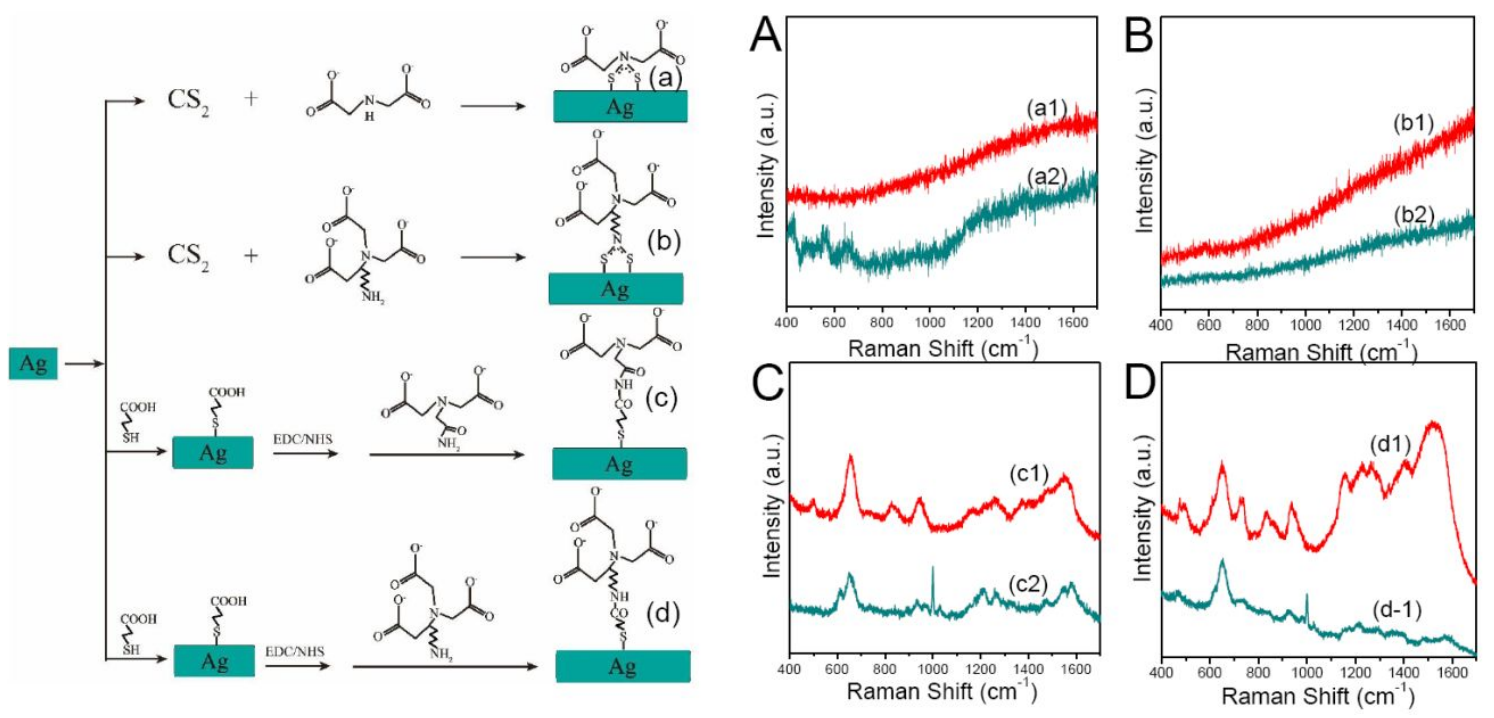

Figure S2. Design and assembly of spacer molecules and the corresponding SERS spectra. Spacers (a) and (b) are based on conventional synthetic route for immobilization of amino groups. In terms spacers (c) and (d), 3-mercaptopropionic acid (MUA) self-assemble layer was used and activated with EDC/NHS, and the (2-acetamido) iminodiacetic acid, $\mathrm{N}_{\alpha}, \mathrm{N}_{\alpha}$-bis(carboxymethyl)-L-lysine hydrate, $\mathrm{Ni}^{2+}$ and the peptides were subsequently immobilized. HF was used as a model protein peptide. (A-D) SERS spectra recorded on the Ag substrate modified with Spacer a-d with $\mathrm{Ni}^{2+}$ (red) and $\mathrm{Ni}^{2+} \& \mathrm{HF}$ (green), respectively.
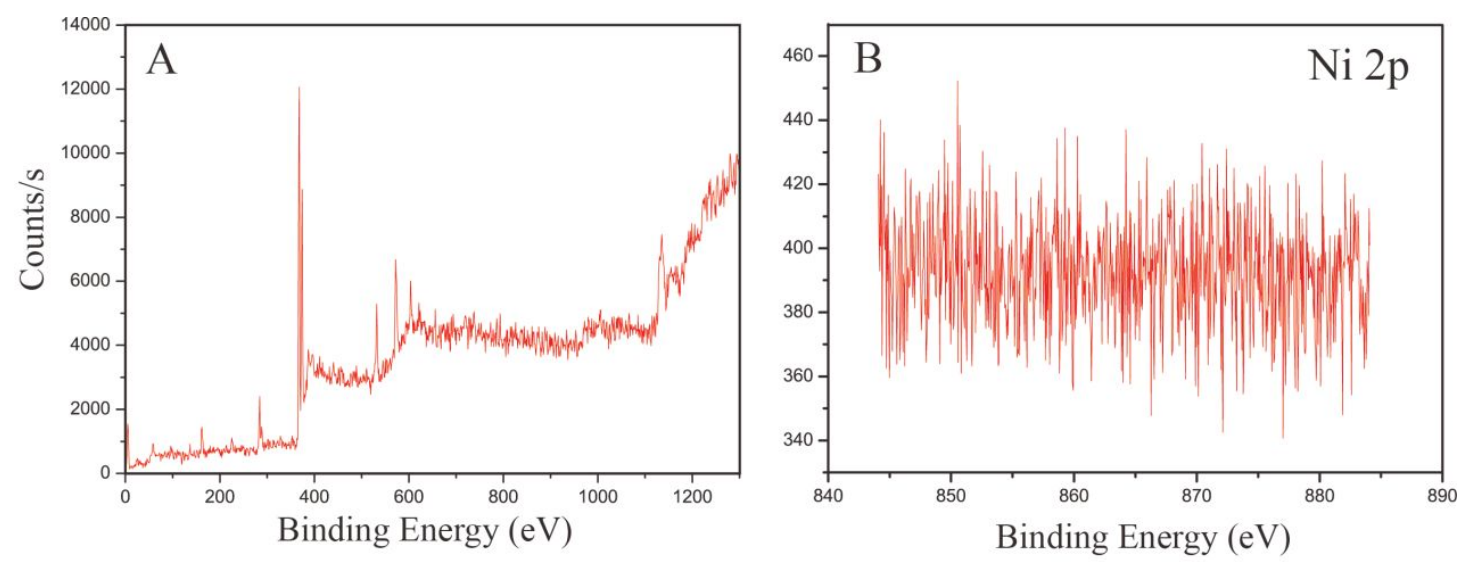

Figure S3. XPS spectra of Spacer a-modified Ag with $\mathrm{Ni}^{2+} \& \mathrm{HF}$ : (A) full-range; (B) Ni 2p. 

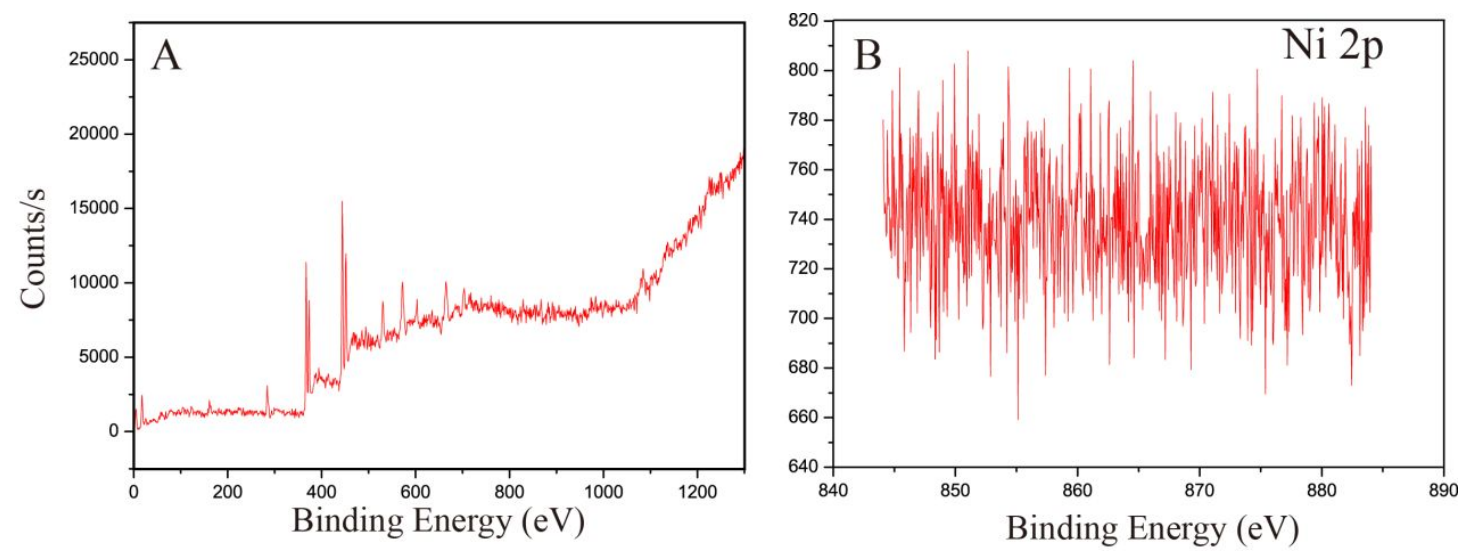

Figure S4. XPS spectra of spacer b-modified Ag with $\mathrm{Ni}^{2+} \& \mathrm{HF}$ : (A) full-range; (B) Ni $2 \mathrm{p}$.

As illustrated in Figure S4 and S5, we cannot find the peak of Ni atoms in Spacer a and b-modified Ag substrate at $854 \mathrm{eV}$, which is consistent with the results of the SERS spectra in Figure S2 A and B. They are also demonstrated that these functionalizations are not suitable for SERS detection of proteins.
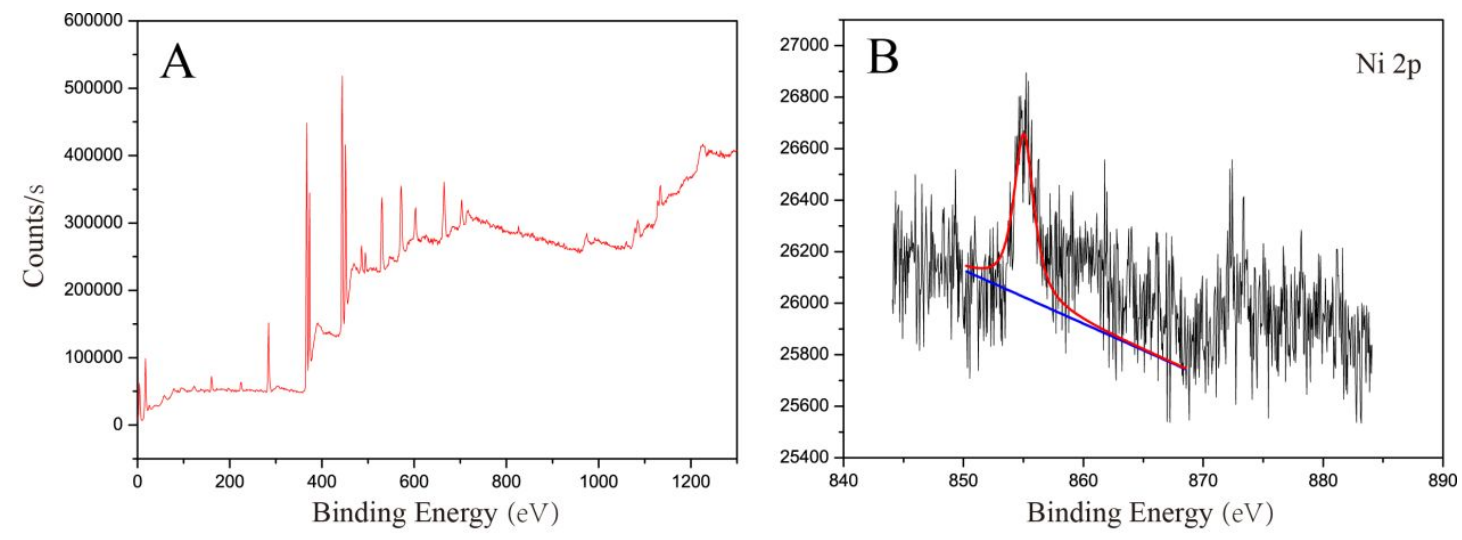

Figure S5. XPS spectra of spacer c-modified Ag with $\mathrm{Ni}^{2+} \& \mathrm{HF}$ : (A) full-range; (B) Ni 2p. 

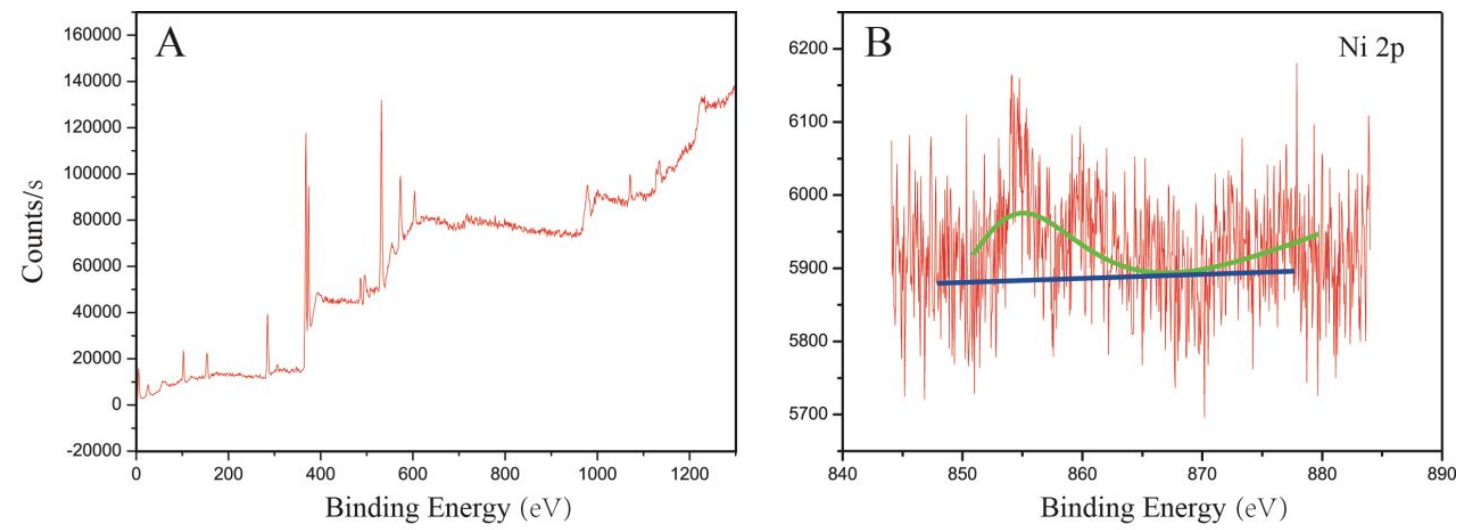

Figure S6. XPS spectra of spacer d-modified Ag with $\mathrm{Ni}^{2+} \& \mathrm{HF}$ : (A) full-range; (B) Ni 2p.
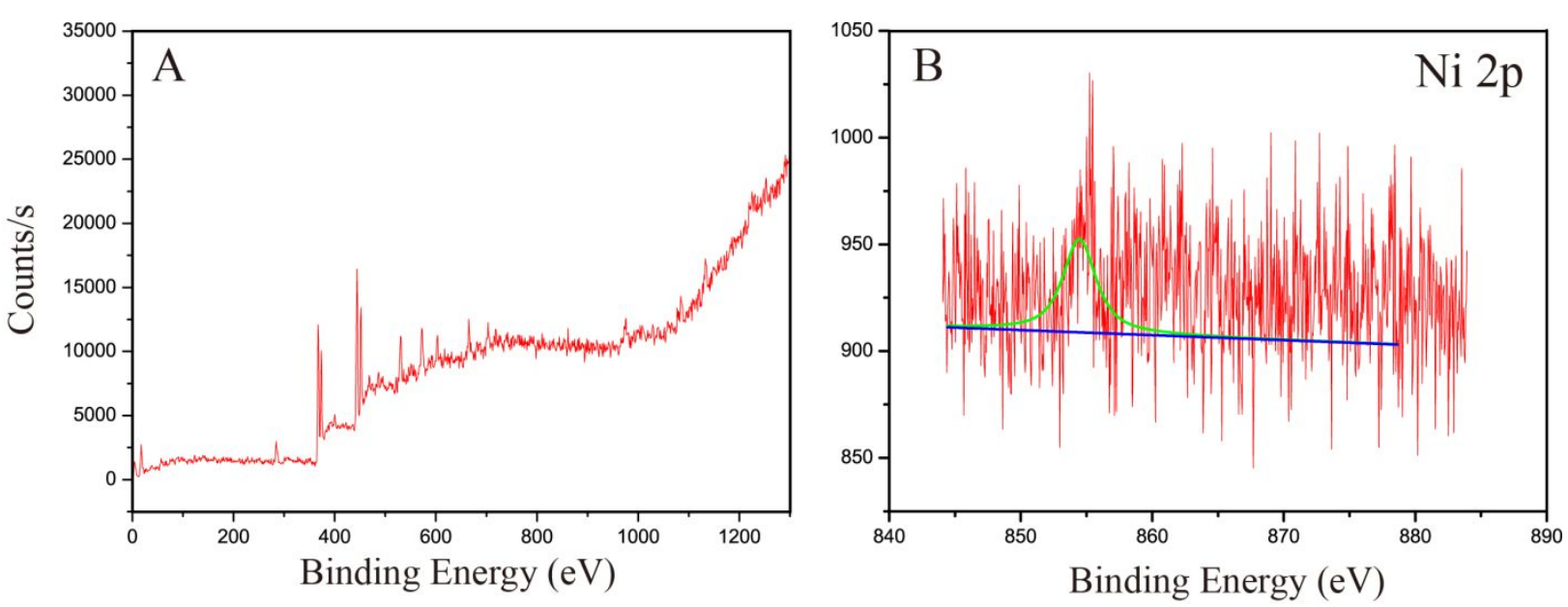

Figure S7. XPS spectra of spacer e-modified Ag with $\mathrm{Ni}^{2+} \& \mathrm{HF}$ : (A) full-range; (B) Ni 2p.

As illustrated in Figure $\mathrm{S} 5 \rightarrow 7$, we can find the peak of Ni atoms in Spacer c, d and e-modified Ag substrate at $854 \mathrm{eV}$, It is very interesting that only one SERS peak located at $1000 \mathrm{~cm}^{-1}$ was observed in both Figure S2 c2 and d2, which is possibly ascribed to the ring breathing vibration of phenyl ring in phenylalanine of HF, indicating that such modifications are not good enough for SERS detection of proteins. 


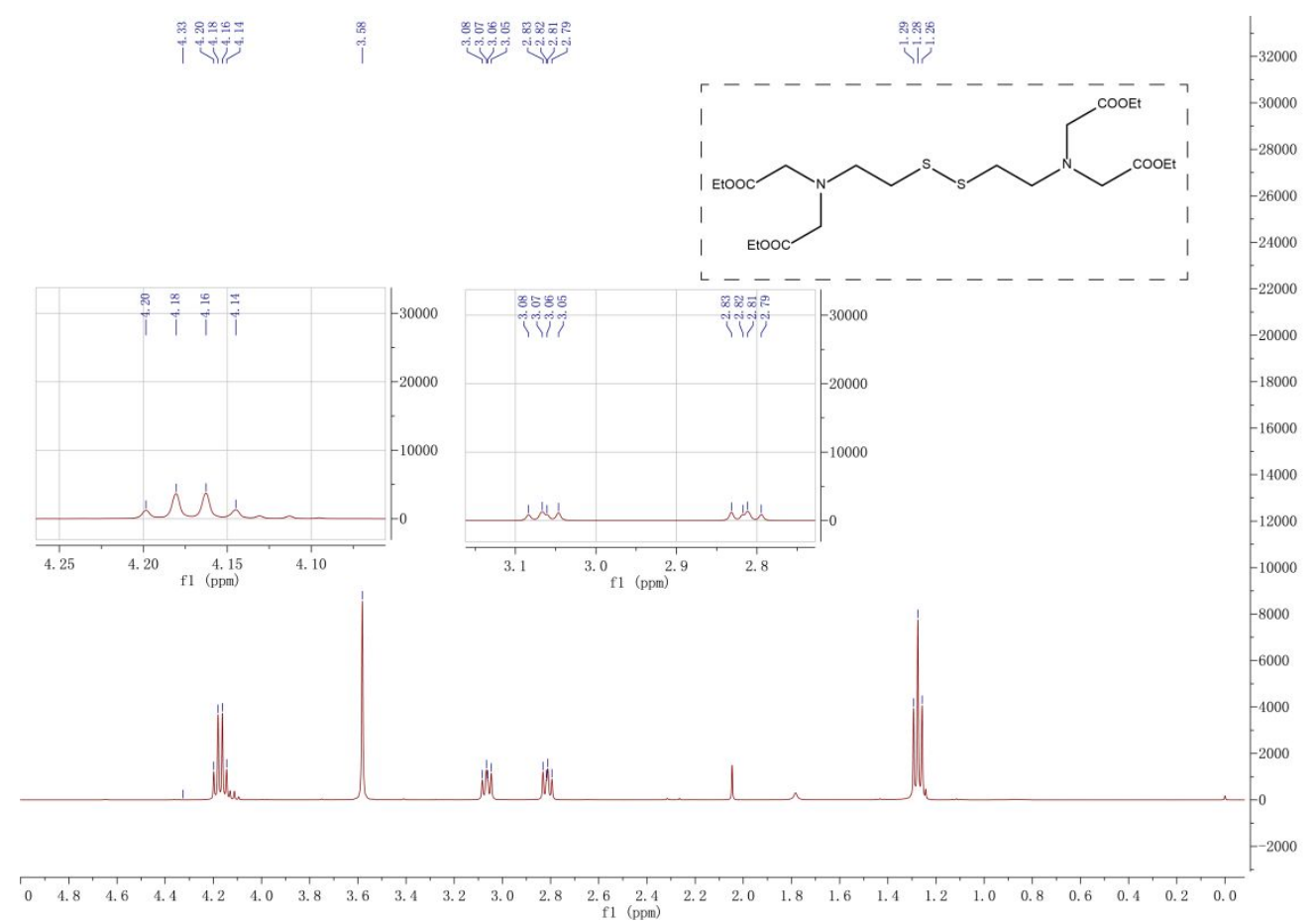

Figure S8. ${ }^{1} \mathrm{H}$ NMR $\left(500 \mathrm{MHz}, \mathrm{CDCl}_{3}\right)$ of compound 1 .

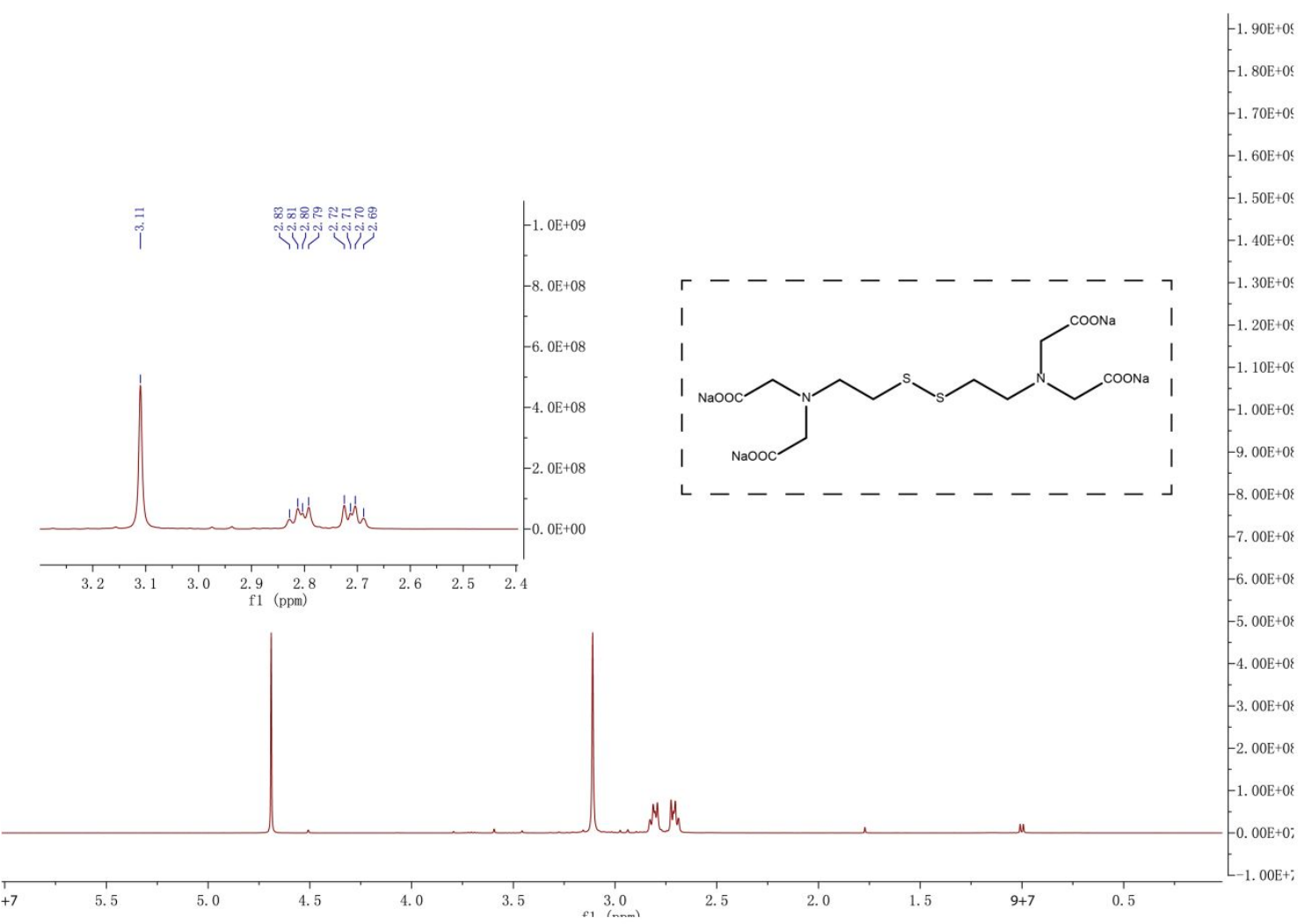

Figure S9. ${ }^{1} \mathrm{H}$ NMR $\left(500 \mathrm{MHz}, \mathrm{D}_{2} \mathrm{O}\right)$ of compound 2 . 
The band around $1660 \mathrm{~cm}^{-1}$ was generally assigned to $\mathrm{C}=\mathrm{O}$ stretching (amide $\mathrm{I}$ ), ${ }^{[4]}$ but the $\mathrm{C}=\mathrm{O}$ stretching vibrations are located beyond $1700 \mathrm{~cm}^{-1}$ according to our DFT results, thus we deduced that the $\mathrm{N}-\mathrm{H}$ vibrations may be associated with amide I band. It is well known that asparagine has one more amino group compared with glycine. Therefore, if $\mathrm{N}-\mathrm{H}$ vibrations are associated with Amide I band, a new tensor would emerge around the Amide I band. As shown in Figure 5, we observed two peaks located at 1611 and $1649 \mathrm{~cm}^{-1}$ from the experimental results of both $\mathrm{HG}$ and $\mathrm{HN}$. Noted that the theoretical tensor of amino group of HG located between the two tensors of the $\mathrm{HN}$, therefore we deduced that beside $\mathrm{C}=\mathrm{O}$ stretching vibrations, the amino groups also have contributed to the Amide I. It is worth noting that the $\mathrm{C}=\mathrm{O}$ stretching vibrations was observed in only one case (Figure 4A) among the six kinds of peptides (Table 1), indicating most of the carboxyl groups are far from the Ag substrates where the SERS signals are masked or weakened. Taken together, we concluded that $-\mathrm{NH}_{2}$ vibrations are also associated with Amide I band, contributed by arginine or/and asparagine residues.

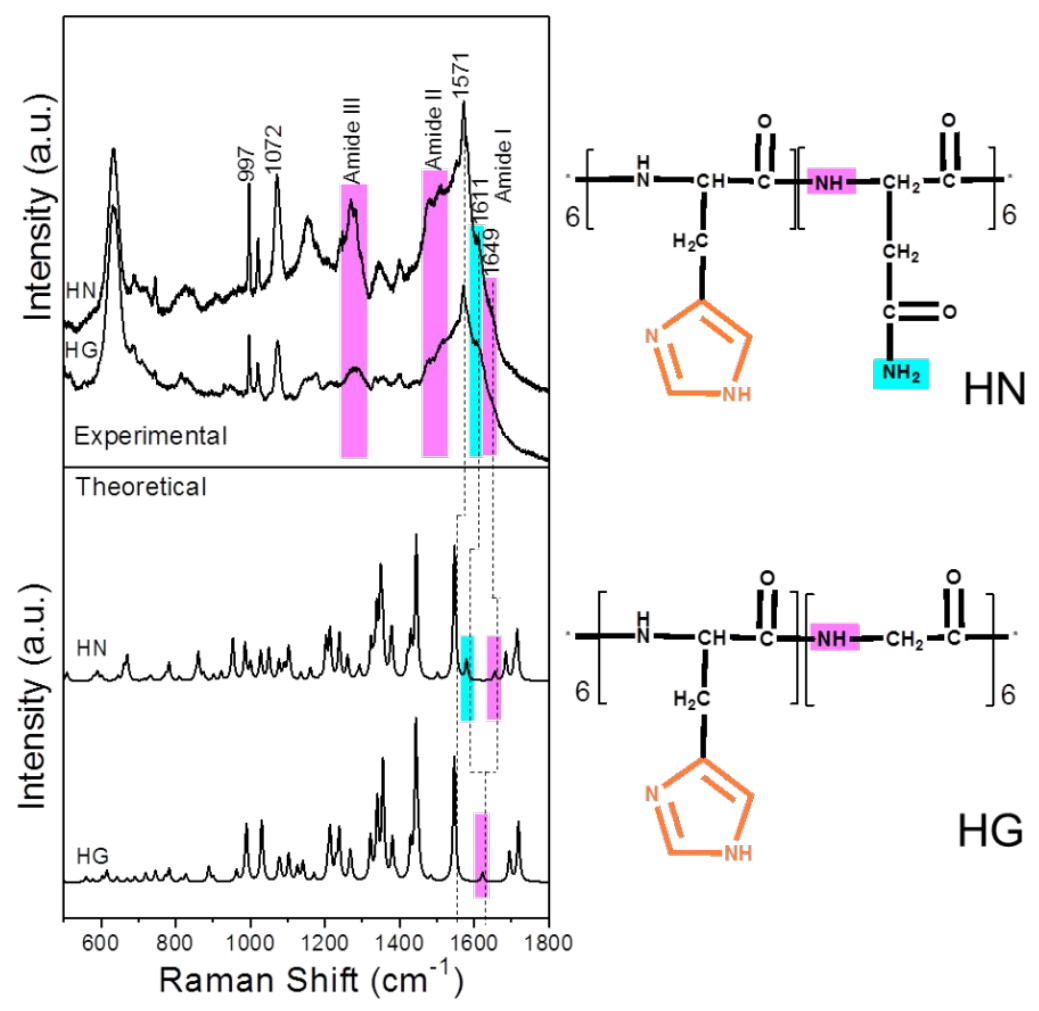

Figure S10. DFT theoretical and experimental SERS spectra of HN and HG. 


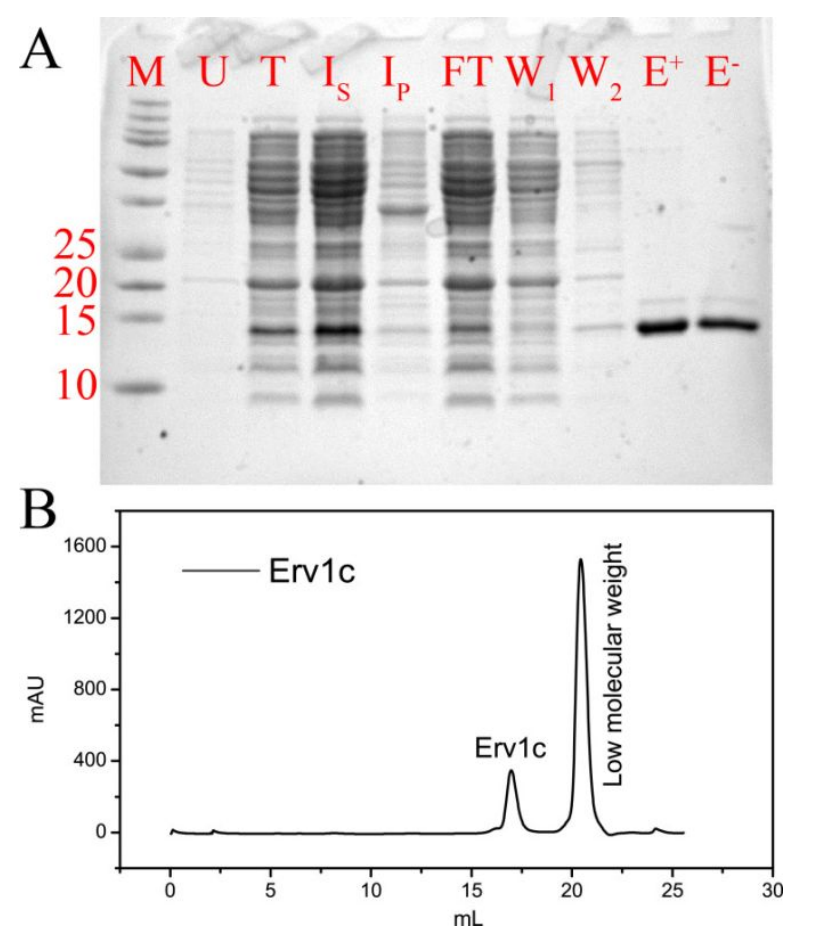

Figure S11. Tricine SDS-PAGE of Erv1c affinity purification visualized using Coomassie-blue staining (A) and (B) size exclusion chromatography of Erv1C.

The reduction of Cyt c can be followed by its color change or absorbance at $550 \mathrm{~nm}$. Here, we followed color change of the Cyt c. As shown in Figure S11, the color of II to V has changed obviously, which indicated the reduction of Cyt c. Here DTT can also reduce Cyt c directly after a longer time (19 VS $2 \mathrm{~min}$ ). Thus, if the reaction time is significantly shortened in the mixture of DTT-Erv1C-Cyt c, the catalytic ability of eluted Erv1C can be confirmed. As shown in Figure S11, both of III and V finished in a shorter time, which indicated the Erv1c in elution preserved catalytic property.

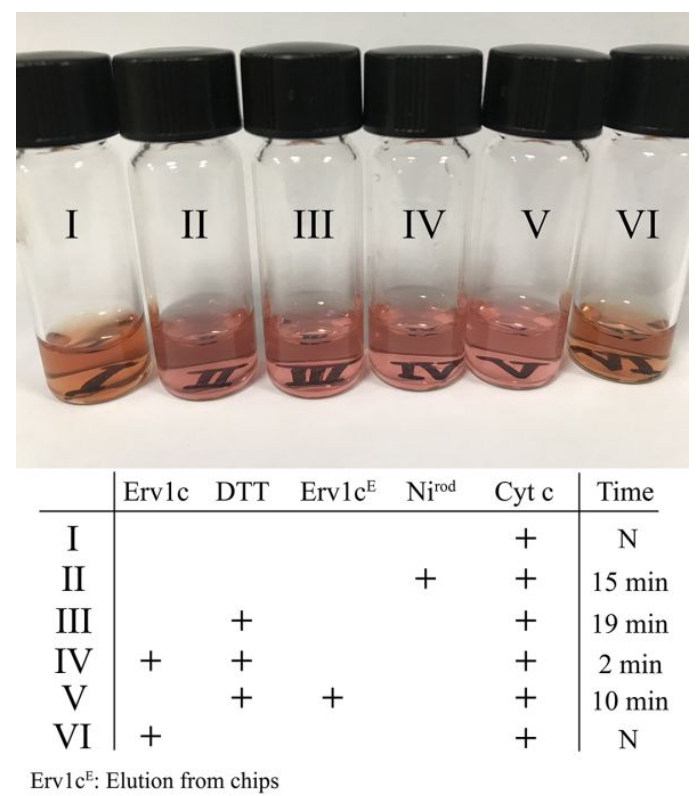

Figure S12. Catalytic properties of the elution of Erv1C from the IDA-modified chips. Erv1C (3 $\mu \mathrm{M})$, Cyt c $(100 \mu \mathrm{M})$ and DTT $(1 \mathrm{mM})$. 


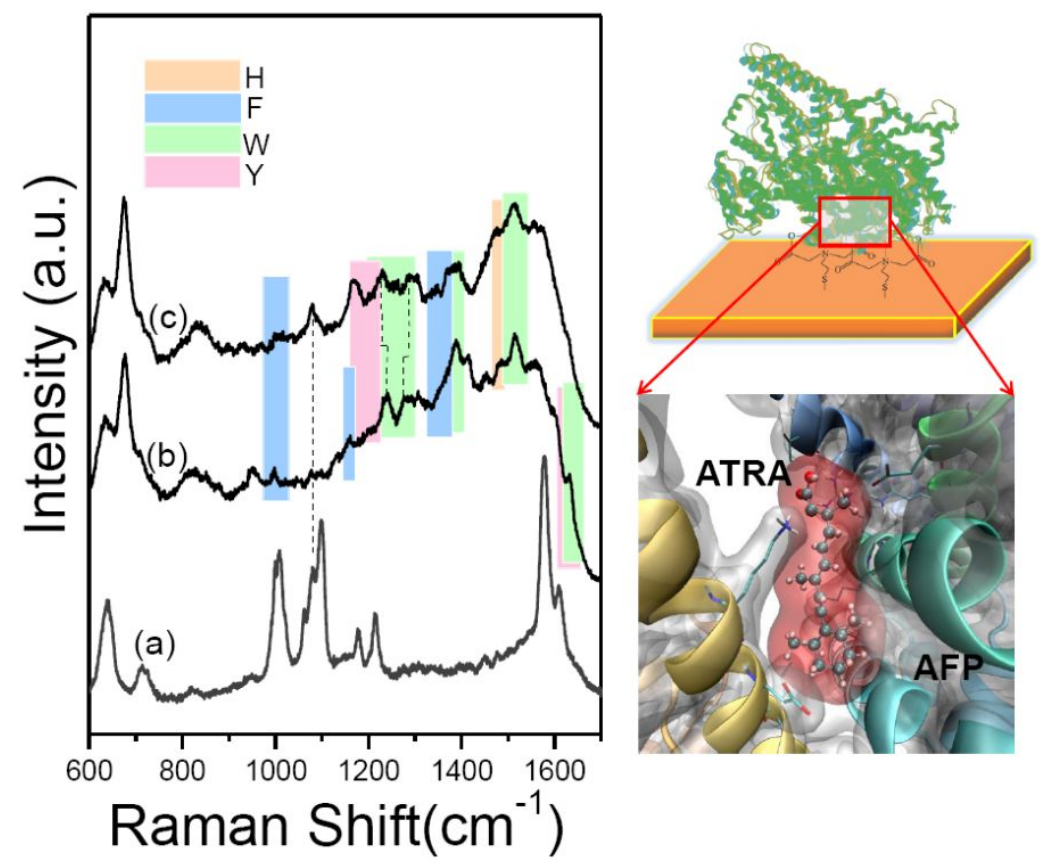

Figure S13. Investigation of AFP-ATRA interactions using IDA-SS. SERS spectra of (a) ATRA, (b) AFP and (c) AFP with ATRA on the IDA-modified Ag substrates.

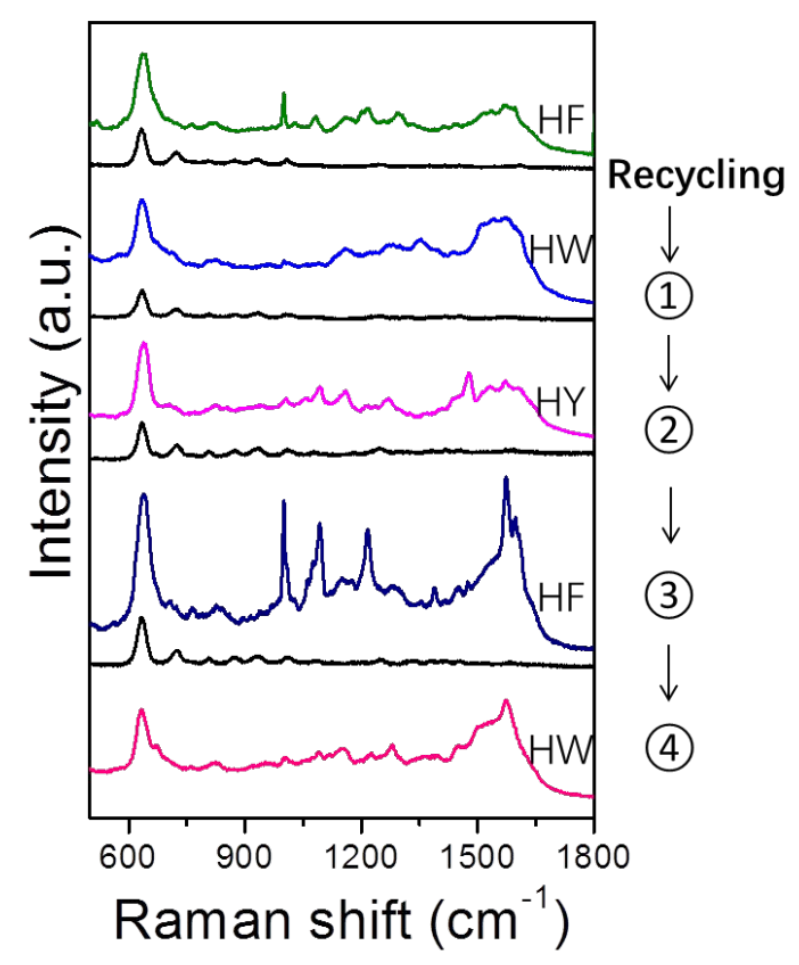

Figure S14. Recycling the IDA-modified Ag substrate for 4 times. SERS spectrum of HF followed by those SERS spectra of 4 His-tagged peptides one after another. 


\section{References:}

(1) Zhu, H.; Coleman, D. M.; Dehen, C. J.; Geisler, I. M.; Zemlyanov, D.; Chmielewski, J.; Simpson, G. J.; Wei, A. Assembly of Dithiocarbamate-Anchored Monolayers on Gold Surfaces in Aqueous Solutions, Langmuir 2008, 24, 8660-8666.

(2) Wasserberg, D.; Cabanas-Danes, J.; Prangsma, J.; O’Mahony, S.; Cazade, P.; Tromp, E.; Blum, C.; Thompson, D.; Huskens, J.; Subramaniam, V.; Jonkheijm, P. Controlling Protein Surface Orientation by Strategic Placement of Oligo-Histidine Tags, ACS Nano, 2017, 11(9), 9068-9083.

(3) Ceh-Pavia, E., Ang, Swee K., Spiller, Michael P., Lu, H. The disease-associated mutation of the mitochondrial thiol oxidase erv1 impairs cofactor binding during its catalytic reaction. Biochem. J. 2014, 464, 449-459.

(4) Lord, R. C.; Yu, N. T. Laser-Excited Raman Spectroscopy of Biomolecules. Journal of Molecular Biology, 1970, 50, 509-524. 